\title{
Influencia de las prácticas de gobierno corporativo y gestión en la percepción de la unidad y armonía en empresas familiares
}

FECHA DE RECEPCIÓN: 27 de julio

FECHA DE APROBACIÓN: 26 de agosto

pp. 159-174

DOI: http://dx.doi.org/10.21158/01208160.n81.2016.1558

\section{Resumen}

El presente artículo hace parte del resultado de la investigación «Ética, Responsabilidad Social y Desarrollo», al partir de una revisión de literatura y de la experiencia previa de los autores. Se identificaron 47 prácticas de gobierno y gestión que pueden incidir en la percepción de unidad y armonía. En una muestra de empresas mexicanas y colombianas se aplicó un análisis de regresión logística donde se encontró que buscar consejo para gestionar el patrimonio, formación continua de líderes, tener accionistas formados, tener estrategia familiar, realizar asambleas de accionistas anualmente y reuniones adicionales de propietarios son las prácticas que mayor influencia tienen en la percepción de unidad y armonía en la empresa familiar.

\section{Palabras clave}

Gobierno corporativo, prácticas de gobierno corporativo, prácticas de gestión, empresa familiar, unidad y armonía.

\section{Gonzalo Eduardo Gómez Betancourt}

PhD. en Management, IESE Business School, Universidad de Navarra; MBA Executive, INALDE Business School, Universidad de La Sabana; Ingeniero Industrial, Pontificia Universidad Javeriana. Es CEO en Legacy and Management Consulting Group.

José Bernardo Betancourt Ramírez

PhD. (c) en Administración Estratégica de Empresas, Centrum Graduate Business School, Pontificia Universidad Católica -Perú-. DBA (c) en Maastricht School of Management -Holanda-. MBA, Universidad Internacional Iberoamericana -Puerto Rico-. MDEEF, Universidad de León -España-. Administrador de Instituciones de Servicio, Universidad de La Sabana. Miembro del grupo de investigación Empresa Familiar, Inalde Business School, Universidad de La Sabana. Director Administrativo, lymgroup.com -Colombia-.

\section{Diógenes Lagos Cortés}

PhD. (c) en Ciencias Administrativas, Universidad Nacional de La Plata -Argentina-; magíster en Ingeniería Industrial, Universidad Industrial de Santander. Investigador del grupo Empresa Familiar, Inalde Business School, Universidad de La Sabana.

\section{Ricardo Aparicio Castillo}

Magíster en Cultura y Gobierno de las Organizaciones, Universidad de Navarra; MBA en Dirección de Empresas, Instituto Panamericano de Alta Dirección de Empresa (IPADE); Ingeniero Mecánico, UNAM, -México-. Profesor de Factor Humano y Empresa-Familia, y director del Centro de Investigación para Familias de Empresarios, IPADE Business School. 


\title{
Influence of Corporate Governance and Management Practices on the Percpetion of Unity and Harmony in Family- owned Companies
}

\begin{abstract}
Abstrac. Starting with a bibliographic review and the former experience from different authors, 47 practices of corporate governance and management were identified which could definitely influence on company unity and harmony. Based on a sample taken of Mexican and Colombian companies, an analysis of logistic regression was applied in which it was found that looking for an advice to manage equity, to have continuing education of leaders, to own a family strategy and to organize shareholders' meetings are the most influential practices to get a perception of unity and harmony in family-owned companies.
\end{abstract}

Key words. Corporate governance, corporate governance practices, management practices, family- owned companies, unity and harmony.

\section{Influence des pratiques de gouvernance et de gestion corporative sur la perception d'unité et d'harmonie des entreprises familiales}

Resumé. A partir d'une analyse de la littérature existante et grâce à l'expérience préalable des auteurs de cet article, nous avons identifié 47 pratiques de gouvernance et de gestion entrepreneuriale pouvant influer sur la perception d'unité et d'harmonie des entreprises familiales. L'étude d'un échantillon d'entreprises mexicaines et colombiennes nous permettra de réaliser une analyse de régression logistique montrant que la gestion du patrimoine, la formation continue des dirigeants, la formation des actionnaires, une stratégie familiale solide, la réalisation d'assemblées annuelles d'actionnaires et des réunions fréquentes des propriétaires sont les pratiques influençant le plus la perception d'unité et d'harmonie des entreprises familiales.

Mots clefs. Gouvernance corporative, pratiques de gouvernance corporative, gestion d'entreprise, entreprise familiale, unité et harmonie.

\section{Influência das práticas de governo corporativo e gestão na percepção da união e harmonia em empresas familiares}

Resumo. A partir de uma revisão da literatura e da experiência dos autores, identificaram-se 47 práticas de governo e gestão, que possam ter um impacto na percepção de União e Harmonia. Em uma amostra de empresas mexicanas e colombianas, aplicou-se uma análise de regressão logística, onde se encontrou que procurar conselho para a gestão do patrimônio, a formação contínua de líderes, ter acionistas formados, ter uma estratégia familiar, realizar assembleias de acionistas anualmente e reuniões adicionais de proprietários, são as práticas de maior influência na percepção de União e Harmonia na empresa familiar.

Palabras chave. Governo corporativo, práticas de governo corporativo, práticas de gestão, empresa familiar, unidade e harmonia. 


\section{Introducción}

T a combinación de los sistemas familia y Uempresa hacen de la empresa familiar un ambiente propicio para el surgimiento de problemas que pueden afectar su supervivencia (Kellermanns \& Eddleston, 2007). Para enfrentar los problemas que afectan la sostenibilidad de la empresa familiar, los académicos del área han realizado contribuciones importantes en materia de gobierno corporativo y aspectos estructurales de la empresa (Suess, 2014), sin embargo, son pocos los estudios empíricos que identifiquen estas dificultades, para analizarlas y proponer soluciones efectivas.

Gallo (1995), describió las trampas familiares, una serie de malos hábitos característicos de las empresas familiares que afectan directamente su cultura. Los conceptos del modelo teórico de las trampas familiares inspiraron la realización de este estudio empírico, que busca identificar cuáles son las prácticas de gobierno y gestión que pueden influir en su unidad y armonía familiar.

Este trabajo se ha estructurado en cinco apartados precedidos por esta introducción. En el segundo apartado, se identifican diez factores que propician el conflicto en la empresa familiar y que pueden afectar la unidad y armonía. Posteriormente, se presenta la metodología con la cual se levantó la información y se analizaron los datos. En seguida, se presentan los resultados y la discusión, para finalmente, exponer las conclusiones e implicaciones.

\section{Factores que propician el conflicto en la empresa familiar}

I os sistemas familia, propiedad y empresa son característicos de la empresa familiar (Tagiuri y Davis, 1992); la interacción entre ellos, sumado a los diferentes roles y expectativas de sus miembros son un entorno propicio para el surgimiento de conflictos (Harvey \& Evans, 1994; Kellermanns \& Eddleston, 2007; Olson et al., 2003), que reducen la efectividad de la empresa y minimizan las potenciales ventajas del grupo familiar (Jehn, 1995). A continuación, se presentan diez factores relacionados con la existencia de conflictos que pueden afectar la unidad y armonía en la empresa familiar.

- Orgullo de los empresarios familiares. Gallo (1995), plantea que a los empresarios familiares que alcanzan cierto prestigio se les dificulta admitir el uso de malas prácticas corporativas, por lo cual prefieren ocultarlas y no pedir consejo a terceros; de esta forma, se retrasa la oportunidad de trabajar en las debilidades que hacen vulnerable a la empresa.

- Ejercer responsablemente la propiedad. Aronoff y Ward (2002), señalan que la familia debe ejercer responsablemente la propiedad. Por ejemplo, ser accionista no implica ser un buen líder, tampoco que se tengan las capacidades y conocimientos directivos necesarios (Gallo, 1995). Los miembros de la familia propietaria deben ejercer 
un adecuado rol como propietarios en la asamblea general de accionistas, aun cuando no participen directamente de la junta directiva o cuando no trabajen en la empresa (Gómez-Betancourt, 2005). De acuerdo con Zahra (2003), el efecto de la propiedad familiar no debe confundirse con el involucramiento delafamilia, un buen propietario puede participar constructivamente en la empresa familiar de distintas formas, por ejemplo, fomentando la alineación de objetivos entre los demás propietarios familiares (Pieper, Klein \& Jaskiewicz, 2008).

- Generación y reparto del valor económico. Gallo (1995) y Gómez-Betancourt (2005), destacan que en las empresas familiares existen prácticas no adecuadas en la generación y el reparto del valor económico, por ejemplo, pagar salarios u honorarios por encima o por debajo de la remuneración del mercado; no pagar al Estado los impuestos que corresponden, usando doble contabilidad o gastos inflados; tener condiciones preferenciales para clientes o proveedores de la familia; y mezclar los activos de la familia con los activos de la empresa, entre otras.

- Uso de órganos de gobierno. El gobierno corporativo se encarga de regular las relaciones de la empresa con la familia, además de definir el rol que desempeñan los miembros familiares en la estructura organizacional (Brenes, Madrigal y Requena, 2011).

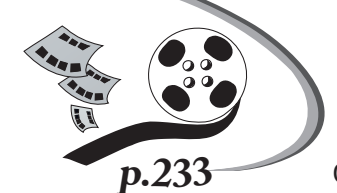
La importancia de la junta directiva en los resultados empresariales y la continuidad de las empresas ha sido destacada en diferentes investigaciones (Daily \& Dalton, 1993; Lester \& Cannella, 2006). Aunque en las empresas familiares existe la tendencia de incorporar miembros independientes en la junta directiva y de realizar asambleas de accionistas y comités formales, es frecuente que los empresarios tiendan a implementar solo lo exigido por la ley.

- Diseño y comunicación de la estrategia. La junta directiva es clave en el desarrollo de la planeación estratégica (Chrisman, Chua y Litz, 2004), en la supervisión del desempeño empresarial y de los gerentes, además de apoyar con su experiencia el curso de la empresa (Schulze, Lubatkin, Dino\& Buchholtz, 2001). Sin embargo, es común encontrar empresas familiares, donde la estrategia es de conocimiento solo del líder, es decir, no se comunica formalmente al resto de colaboradores, por lo tanto, no se trabaja periódicamente en ella, ni se controla su implementación. De otra parte, la inconsistencia entre valores y metas, recursos y capacidades, entorno, estructura y sistemas organizativos de la empresa pueden influir en el éxito de la estrategia (Grant, 2016).

- Diseño de estructuras organizativas. La estructura de la organización debe estar diseñada de acuerdo con la estrategia que se busca implementar (Chandler, 1962; Galbraith, 1995). Sin embargo, en las empresas familiares, a menudo, se crean cargos para los mismos miembros de la familia que no tienen la formación o la experiencia necesaria para desempeñar con éxito sus trabajos (Fiegener, Brown, Prince\& File, 1996), ya que es usual que en sus estructuras organizativas prime más el árbol genealógico que una estructura basada en la estrategia; con ello pierden competitividad (GómezBetancourt, 2005). Esto puede generar que los empleados cualificados dejen o eviten estas empresas debido al limitado potencial de crecimiento profesional (Sirmon \& Hitt, 2003). 
- Uso de sistemas de dirección. El uso de mecanismos de dirección, como la evaluación, compensación, información, comunicación, y planes de carrera ayudan a los directivos a lograr que los miembros de la organización se enfoquen en alcanzar los objetivos propuestos, así como a comprometerse en el largo plazo. En este sentido, es importante la distribución de responsabilidades y la asignación de recursos que contribuyan a la creación de un buen ambiente societario y laboral (GómezBetancourt, 2010). De igual forma, la junta directiva debe evaluar formal y regularmente al gerente general, a los ejecutivos principales y a la misma junta directiva con relación al cumplimiento de los objetivos organizacionales y el desempeño (Lane, Astrachan, Keyt \& McMillan, 2006).

- Valores familiares. Los valores familiares son un elemento fundamental para dar continuidad a un legado (Gómez-Betancourt, 2010). La pérdida de estos está relacionada con cambios en la cultura y la tolerancia frente a aspectos que terminan validando acciones poco éticas y afectando a la familia, la empresa e incluso el patrimonio. Es el caso de las relaciones extramatrimoniales, p.233 vincular el cierre de negocios
el alcohol, disfrutar el dinero hasta caer en la opulencia, evadir el pago de impuestos con la excusa de la corrupción estatal, entre otros.

- Sucesión. De acuerdo con Hoekman (1999), más del 70 \% de la primera generación de fundadores de empresas familiares no hacen un plan de sucesión. En la empresa familiar se debe realizar la transmisión del patrimonio, la cesión del liderazgo a un nuevo gerente y a un nuevo líder familiar, pues cualquiera de ellas tiene implicaciones económicas, legales, y sobre todo, emocionales, tanto para el fundador, como para los sucesores. Por esta razón, se tiende a posponer cualquier decisión al respecto (Lansberg, 1999; Ward, 2011), cuando debería ser discutida y planeada con la familia, los potenciales propietarios y la junta directiva en el caso de la sucesión ejecutiva.

- Riesgos relacionados con la violencia y la inseguridad. En países como Colombia y México existen problemas de violencia, las familias propietarias deben enfrentar amenazas, robos, extorsiones e incluso secuestros. Una investigación realizada por Londoño (1996), reveló que el 4 \% de los entrevistados tuvo un familiar secuestrado; ante este tipo de contingencias la familia debe tener un criterio unificado para gestionar la situación y prevenir discusiones que puedan generar el rompimiento de la unidad familiar.

Este estudio empírico tiene como objetivo identificar las prácticas de gobierno corporativo y gestión que influyen en la unidad y armonía de la empresa familiar, para ello, se planteó la siguiente hipótesis:

Algunas prácticas de gobierno corporativo y gestión implementadas en la empresa familiar influyen en su unidad y armonía. 


\section{Metodología}

\subsection{Datos y muestra}

Se aplicó un muestreo por conveniencia en estudiantes y egresados de dos escuelas de negocios (Inalde Business School, Colombia e IPADE Business School, México). En el caso colombiano la encuesta fue también dirigida a los lectores de la revista digital de negocios, Dinero.com. En total la encuesta fue respondida por 620 personas, no obstante, para el análisis, se usaron solamente las encuestas con respuestas completas de accionistas, miembros de junta directiva, directivos y miembros de la familia propietaria. La muestra final se compone de 285 encuestas, 75 en Colombia y 210 en México.

\subsection{Modelo de investigación}

\subsubsection{Variable dependiente}

Unidad y armonía. Este concepto surge de la propuesta de Gómez (2000), que combina dos elementos: i) unidad familiar, entendido como la capacidad de comunicación y cohesión entre los miembros de la familia, y que puede estar apoyada en procesos y estructuras formales (Peterson \& Distelberg, 2011); y ii) armonía familiar, visto como la capacidad de crear y mantener buenas relaciones y confianza entre los miembros de la familia (Jiménez, Martos y Jiménez, 2015; Li, 2006). La encuesta indagó acerca de la percepción que el encuestado tenía con respecto a la unidad y armonía de la familia en los últimos cinco años, mediante una escala tipo Likert con los siguientes valores: (1) (ha empeorado notablemente); (2) (ha empeorado); (3) (no ha cambiado); (4) (ha mejorado); y (5) (ha mejorado notablemente).

\subsubsection{Variables independientes}

Prácticas de gobierno y gestión. Tomando como base los diez factores identificados en la revisión de literatura y la experiencia previa de los autores, se definieron 47 prácticas relacionadas con los sistemas: empresa, familia y propiedad (Tabla 1), que pueden influir en la unidad y armonía de la empresa familiar. La percepción ${ }^{1}$ acerca de dichas prácticas se indagó en la encuesta donde fueron redactadas con sentido positivo, a manera de buena práctica, o negativo, como una mala práctica, para evitar sesgos en las respuestas por parte de los encuestados. Para ello, se usó una escala tipo Likert con los siguientes valores: (0) (no sabe, no responde); (1) (totalmente en desacuerdo); (2) (en desacuerdo); (3) (ni de acuerdo ni en desacuerdo); (4) (de acuerdo); y (5) (totalmente de acuerdo).

\subsubsection{Variables de control}

Se incorporó al análisis los siguientes controles: edad del líder, género del líder, líder familiar o No, generaciones en la empresa $-1^{\circ}, 2^{\circ}, 3^{\circ}$, $4^{\circ}-$, sector -industria, servicios, otros sectores-; tamaño de la empresa -micro, pequeña, mediana, grande-; rentabilidad patrimonial y país -Colombia, México-.

\footnotetext{
1 Investigaciones anteriores en el campo de la empresa familiar han usado las percepciones desde el concepto de cognición colectiva, entendido como el grado en que los familiares creen que la unidad del grupo existe y se convierte en la base para obtener resultados positivos que motivan la acción conjunta (Habbershon \& Astrachan, 1997). De acuerdo con Corbella (1994), con las percepciones, los encuestados extraen de forma automática e inconsciente la información, esto hace que sean una alternativa frente al problema de medición de fenómenos psicosociales, donde el interés del investigador se enfoca en propiedades que no son observables (Mora y Araújo, 2012). Para Habbershon \& Astrachan (1997), la percepción de un miembro de familia converge con la percepción del grupo, y al capturar la percepción individual se pueden capturar conocimientos y creencias colectivas en un momento específico de tiempo.
} 
Tabla 1. Resumen de prácticas de gobierno corporativo y gestión en empresas familiares.

Orgullo de los empresarios familiares

(1) El líder de la empresa considera necesario el consejo de otros para el buen desarrollo de la empresa.

(2) La persona que lidera el patrimonio considera necesario el consejo de otros para el buen desarrollo del patrimonio familiar.

(3) La persona que lidera la familia considera necesario el consejo de otros para el buen desarrollo de la unidad y armonía familiar.

(4) En los últimos cinco años se han contratado asesores de empresa familiar.

Ejercer responsablemente la propiedad

(5) Los líderes asisten periódicamente a programas de formación directiva, conferencias y talleres, para el buen desarrollo de sus funciones.

(6) Los actuales y potenciales accionistas familiares tienen los conocimientos necesarios para ejercer un rol responsable como propietarios.

(7) Cada cargo en la empresa tiene niveles de autonomía en la toma de decisiones, responsabilidades y perfil del cargo claramente definido.

Generación y reparto del valor económico

(8) Los miembros familiares que son clientes o proveedores de la empresa reciben igual trato que los demás.

(9) Se remunera el trabajo de los miembros familiares a valor del mercado al igual que a otros trabajadores.

(10) Los accionistas usan los recursos de la empresa para uso personal.

(11) La empresa paga los impuestos que le corresponden.

(12) Existen negocios de familiares vinculados a la empresa sin la suficiente claridad del manejo de los flujos económicos - descuentos, precios, comisiones, inversiones, etc.-.

Uso de órganos de gobierno

(13) La empresa cuenta con una junta directiva formal y registrada ante cámara de comercio, que se reúne periódicamente a ejercer su rol.

(14) La empresa tiene una junta directiva o consejo asesor con miembros externos independientes que trabajen en la estrategia.

(15) La compañía realiza anualmente una asamblea general de accionistas donde se toman decisiones sobre inversiones, dividendos, endeudamiento, nombramiento de junta directiva, entre otros.

(16) La familia se reúne periódicamente a discutir temas de índole familiar -en algunas familias este espacio de debate se denomina concejo de familia-.

(17) Los propietarios de la familia hacen reuniones adicionales a la asamblea general de accionistas para discutir periódicamente aspectos de la propiedad, analizar información y tomar decisiones.

Diseño y comunicación de la estrategia

(18) La empresa tiene una estrategia explícita para abordar los mercados.

(19) Los propietarios de la familia tienen una estrategia patrimonial explícita donde se establecen porcentajes de diversificación, riesgo, sectores de interés, etc..

(20) Los miembros de la familia tienen una estrategia familiar explícita con actividades que fomentan la unidad y armonía, y que les facilita resolver conflictos.

(21) Los miembros de la familia, especialmente quienes ostentan el poder en la empresa, tienen proyectos de vida.

Diseño de estructuras organizativas

(22) La empresa cuenta con una estructura directiva basada en la estrategia planeada y las necesidades de la empresa.

(23) La familia propietaria cuenta con una estructura tipo holding que le permite gestionar los diferentes elementos del patrimonio familiar -empresas, inversiones, liquidez, finca raíz, etc.-.

(24) Tienen estructuras legales -testamentos, fideicomisos, sociedades nacionales, en el exterior- que les permite planear la sucesión patrimonial, así como hacer transacciones y reparto de la propiedad.

(25) La familia tiene una persona dedicada a fortalecer la unidad y armonía familiar.

Uso de sistemas de dirección

(26) Se tienen sistemas de evaluación de desempeño, que dejan a un lado el carácter familiar de cada cargo directivo, que miden el cumplimiento de la estrategia y las funciones del cargo para que las personas accedan a bonos.

(27) La participación en los órganos de gobierno es remunerada y evaluada -ej.: junta directiva, consejo asesor, consejo de socios, consejo de familia-.

(28) La persona que maneja los temas del patrimonio familiar recibe remuneración por su labor y es evaluada con respecto a la evolución del patrimonio. 
Tabla 1. Resumen de prácticas de gobierno corporativo y gestión en empresas familiares (Continuación)

(29) La persona que se encarga de mantener a la familia unida y en armonía recibe remuneración por su labor y es evaluada en términos de cómo va la familia y sus valores.

(30) El revisor fiscal visita la empresa periódicamente, informa irregularidades a la junta directiva o a la asamblea general de accionistas, revisa la contabilidad, y las actas, y es remplazado cada cinco años.

(31) Los accionistas reciben información completa y oportuna para la asamblea general de accionistas, y periódicamente, durante el año.

(32) La familia tiene un medio de comunicación por el cual convoca e informa sobre acontecimientos importantes.

Valores familiares

(33) Los miembros de la familia permiten que los conflictos familiares sean conocidos por empleados y terceros, afectando así el desarrollo de la estrategia empresarial.

(34) En la empresa, los directivos y trabajadores deben actuar de acuerdo con los valores de la familia para permanecer en la empresa y acceder a bonos.

(35) Existen sanciones claras para los familiares, accionistas, directivos o trabajadores que actúen en contra de los valores de la familia.

(36) Algunos miembros de la familia aprovechan su vínculo o su posición para obtener beneficios económicos y de otra índole frente a clientes, proveedores, socios o trabajadores.

(37) Se apoya el emprendimiento en la familia con recursos económicos y guía de un directivo.

Sucesión

(38) Los fundadores han hablado con sus sucesores, escuchado sus expectativas y comentado las aptitudes de cada miembro familiar que serán tenidas en cuenta en el momento de repartir la propiedad.

(39) El líder empresarial tiene más de 65 años y sigue ostentando el poder.

(40) La junta directiva ha seleccionado al sucesor para la gerencia general de la compañía, lo está formando y está en proceso de coaching.

(41) Existen sucesores claros y aceptados para manejar la empresa, la familia y el patrimonio.

(42) El sucesor tiene la capacidad de manejar la empresa, la familia y la propiedad.

(43) El líder prefiere un sucesor externo a un familiar.

Riesgos relacionados con violencia e inseguridad

(44) Algún miembro de la familia ha sufrido secuestros.

(45) Algún miembro de la familia ha sufrido extorsiones -implica pago de dinero- o amenazas.

(46) Tienen acuerdos para enfrentar situaciones de riesgo a la vida o integridad de un familiar.

(47) Tienen prácticas en la familia para no llamar la atención ni ser objetivo de grupos o terceros al margen de la ley.

Fuente. Elaboración propia de los autores.

Con el objetivo de aplicar un análisis de regresión logística que permita identificar las prácticas de gobierno y gestión que inciden en la percepción de la unidad y armonía familiar, se recodificaron, tanto la variable dependiente como las variables independientes, en variables dicotómicas de la siguiente forma: en el caso de la variable dependiente las opciones de respuesta se agruparon en dos categorías: (i) ha empeorado o se mantiene -valores iniciales 1, 2 y 3-;y (ii) ha mejorado -valores iniciales 4 y 5 -.
De igual forma, en las variables independientes se crearon dos categorías: (i) en desacuerdo -valores iniciales 1, 2 y 3-; y (ii) de acuerdo -valores iniciales 4 y $5-$. Esta técnica fue utilizada debido a que la interpretación de los coeficientes del modelo estadístico se simplifica y facilita la

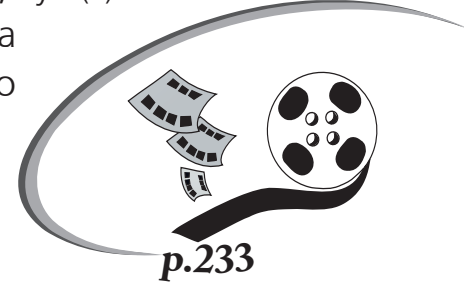
presentación de las relaciones entre las variables independientes y la variable dependiente. 


\section{Resultados}

\subsection{Descripción de la muestra}

Del total de las empresas analizadas, 18,6 \% eran microempresas; $25,6 \%$ eran pequeñas empresas; $28,4 \%$ eran medianas empresas y $27,4 \%$ eran grandes empresas. Con respecto al género del líder, 86,3\% de las empresas eran dirigidas por hombres, y $13,7 \%$ por mujeres, en tanto que el $28,4 \%$ de las empresas eran dirigidas por líderes de la familia y 71,6 \% por personas externos a la familia. Sobre la edad del líder, se encontró que el 4,9\% de las empresas eran dirigidas por personas menores de 35 años, el 43,5\% por personas entre 35 y 55 años, y el $51,6 \%$ por personas mayores de 55 años.

En cuanto a las generaciones de accionistas o directivos de la familia propietaria, se encontró que el 35,8 \% de las empresas era de primera generación; el 47,0 \% estaba en segunda generación; 15,8 \% era de tercera generación, y solo el 1,4 \% de las empresas era de cuarta generación. Con respecto a los sectores, la industria tenía el 61,1\% de las empresas, servicios el 33,0 \%, y el 6,0 \% pertenecían a otros sectores. En cuanto a la rentabilidad patrimonial, se encontró que el 5,6\% de los casos registró rentabilidad negativa; el $62,8 \%$ presentaba rentabilidades entre $0 \%$ y $10 \%$; y finalmente, las empresas con rentabilidades superiores al $10 \%$ eran el 31,6\% de la muestra.

\subsection{Resultados y discusión}

El cuestionario recogía el nivel de acuerdo o desacuerdo de los encuestados frente a 47 prácticas de gobierno y gestión (Tabla 1), identificadas a partir de la revisión de literatura y la experiencia previa de los autores, además de la percepción de la unidad y armonía familiar. Esta información se analizó mediante el procedimiento de regresión logística y se encontró que seis factores son los que más influyen en los dos aspectos mencionados anteriormente (Tabla 2).

Tabla 2. Resultados del modelo de regresión logística.

\begin{tabular}{|c|c|c|c|c|c|}
\hline & B & $\begin{array}{l}\text { Error } \\
\text { estándar }\end{array}$ & Wald & Sig. & $\operatorname{Exp}(B)$ \\
\hline Rentabilidad patrimonial. & 0.720 & 0.266 & 7.316 & 0.007 & 2.054 \\
\hline $\begin{array}{l}\text { (2) Consejo de otros para el buen desarrollo del patrimonio } \\
\text { familiar. }\end{array}$ & 0.871 & 0.329 & 7.004 & 0.008 & 2.389 \\
\hline $\begin{array}{l}\text { (5) Los líderes asisten periódicamente a programas de } \\
\text { formación directiva. }\end{array}$ & 0.789 & 0.300 & 6.904 & 0.009 & 2.201 \\
\hline $\begin{array}{l}\text { (6) Los actuales y potenciales accionistas familiares tienen los } \\
\text { conocimientos. }\end{array}$ & 0.622 & 0.298 & 4.344 & 0.037 & 1.862 \\
\hline $\begin{array}{l}\text { (15) La compañía realiza anualmente una asamblea general } \\
\text { de accionistas. }\end{array}$ & -0.699 & 0.328 & 4.541 & 0.033 & 0.497 \\
\hline $\begin{array}{l}\text { (17) Los propietarios hacen reuniones adicionales a la } \\
\text { asamblea de accionistas. }\end{array}$ & 0.816 & 0.314 & 6.776 & 0.009 & 2.262 \\
\hline (20) La familia tienen una estrategia familiar explícita. & 1.200 & 0.312 & 14.781 & 0.000 & 3.321 \\
\hline País & -1.305 & 0.327 & 15.959 & 0.000 & 0.271 \\
\hline Constante & -2.957 & 0.712 & 17.240 & 0.000 & 0.052 \\
\hline
\end{tabular}

Fuente. Elaboración propia de los autores. 
La combinación de las variables en el modelo arrojó un Ji cuadrado de 80,747 $(p<0,010)$ y $73,3 \%$ de clasificación correcta de los datos. El $R$ cuadrado de Cox y Snell indica que el $24,7 \%$ de la variación de la variable dependiente es explicada por las variables incluidas en el modelo; y el $\mathrm{R}$ cuadrado de Nagelkerke, arroja un 33,2 \% con tres iteraciones y cambios menores a .001.

A continuación, se presentan los hallazgos y se discute su impacto en la unidad y armonía familiar:

- Hallazgo 1. La probabilidad de tener una percepción de mejoramiento en la unidad y armonía es más alta -2.39 veces- cuando la persona que lidera el patrimonio considera necesario el consejo de otros para el buen desarrollo del patrimonio familiar. Lave \& Wenger (1991), plantean que las empresas familiares pueden ser concebidas como organizaciones para el aprendizaje, donde la práctica y la teoría se viven en comunidad. En este sentido, la principal enseñanza para los miembros de una familia propietaria son los valores; la confianza es uno de los principales elementos para construir bases a largo plazo (Gómez, 2000; GómezBetancourt, 2010). Un líder que confía y busca el consejo de expertos transmite un mensaje de humildad a la familia y promueve la búsqueda del conocimiento.

- Hallazgo 2. La probabilidad de tener una percepción de mejoría en la unidad y armonía es más alta -2.20 veces- cuando los líderes asisten periódicamente a programas de formación directiva para el buen desarrollo de sus funciones. En las empresas familiares deben promoverse los acuerdos y buenas prácticas que les permitan afrontar de una mejor manera las diferencias y conflictos de interés que se presentan durante el funcionamiento normal del negocio (Botero, Gomez-Betancourt y Lopez, 2015); por ello, la formación continua de los líderes conlleva a que se identifiquen e implementen planes de acción que faciliten la relación entre los sistemas de la familia y la empresa, y por lo tanto, mejore la unidad y armonía.

- Hallazgo 3. La probabilidad de tener una percepción de mejoría en la unidad y armonía es más alta -1.86 veces-, cuando los actuales y potenciales accionistas familiares tengan los conocimientos necesarios para ejercer un rol responsable como propietarios. Recientes investigaciones han demostrado que las nuevas generaciones en las empresas familiares deben tener la capacidad de asumir varios roles, identidades y oportunidades (Barbera, Bernhard, Nacht \& McCann, 2015). La formación de propietarios es el camino adecuado para que los accionistas tomen decisiones responsables que impacten positivamente la organización y apoyen su estrategia. Un propietario formado conoce el nivel de rentabilidad que debe exigir, la reinversión que debe hacer y los riesgos que asumirá con sus decisiones. El conocimiento puede disminuir los malos entendidos basados en supuestos, y por lo tanto, facilitar que el ambiente familiar mejore.

- Hallazgo 4. La probabilidad de tener una percepción de deterioro en la unidad y armonía es más alta - .50 veces-, cuando la compañía realiza anualmente una asamblea general de accionistas donde se toman decisiones sobre inversiones, dividendos, endeudamiento, nombramiento de junta directiva, entre otros. En algunas empresas familiares, los propietarios tienen una cultura hacia la individualidad (BetancourtGómez, y López, 2011; Jussila, 2006), donde cada accionista quiere manejar sus propios 
recursos. En esta situación se pueden generar fricciones en las relaciones, ya que el accionista delega el manejo de su propiedad y dividendos en una reunión anual en la que se somete a la decisión tomada por la mayoría de votos de accionistas; esto puede generar insatisfacción en el corto plazo. Es importante que la familia propietaria identifique el perfil de propietarios que tiene -individualistas, intermedios o colectivosy luego diseñe políticas para gestionar la propiedad y sus rendimientos. Es posible, que, si se aumenta la frecuencia de las reuniones, la cultura de propietarios se fortalezca y mejore el clima societario, por lo tanto, aumente la unidad y armonía de la familia propietaria.

- Hallazgo 5. La probabilidad de tener una percepción de mejoría en la unidad y armonía es más alta -2.26 veces-, cuando los propietarios de la familia hacen reuniones adicionales a la asamblea general de accionistas para discutir periódicamente aspectos de la propiedad, analizar información y tomar decisiones. Las familias con trayectoria empresarial suelen constituir un portafolio robusto con la empresa familiar de origen, este es diversificado con inversiones en otros negocios, bienes raíces, liquidez, bienes suntuarios (Gómez-Betancourt, 2010), fundaciones familiares e incluso pueden incluir propiedades destinadas para las vacaciones familiares (Jaffe \& Lane, 2004). Con el tiempo terminan implementando estructuras para agrupar las sociedades que pueden o no estar relacionadas con la empresa familiar original. En estas sesiones adicionales se trabajan aspectos relacionados con la diversificación, el riesgo y la rentabilidad (Betancourt, 2013), que le proporcionan al accionista mayor conocimiento, tranquilidad y confianza en los administradores, por lo tanto, se impacta positivamente la unidad y armonía del grupo familiar que interactúa en los negocios.

- Hallazgo 6. La probabilidad de tener una percepción de mejoría en la unidad y armonía es más alta -3.32 veces-, cuando los miembros de la familia tienen una estrategia familiar explícita con actividades que fomentan la unidad y armonía, y que les facilita resolver conflictos. Una familia empresaria puede mantenerse unida a través de una estrategia conjunta que genere una orientación sobre cómo actuar (Betancourt et al., 2011; Jaffe \& Lane, 2004), pues la estrategia familiar permite acordar acciones que fomenten la unidad entre sus miembros, incrementen el sentido de pertenencia a la familia y minimice el efecto negativo de conflictos (Betancourt et al., 2011), además de promover acciones con fines no económicos, como actividades filantrópicas, de ocio, de formación, entre otras.

- Hallazgos relacionados con las variables de control. Se identificó la influencia de la rentabilidad patrimonial en la unidad y armonía, lo que sugiere una relación directa entre la prosperidad económica y las buenas relaciones familiares. La investigación y la teoría se han concentrado en la influencia de la familia en la empresa, y cómo esta relación puede influir en su desempeño económico (Basco y Rodríguez, 2011); sin embargo, no se ha investigado cómo el desempeño económico puede influir en las relaciones familiares. Es importante que en las empresas familiares no se formen inversionistas centrados en intereses económicos, sino que se fomente un rol más responsable de los propietarios (Betancourt, 2013; Gómez-Betancourt y Zapata-Cuervo, 2013), ya que la motivación y el compromiso son un vínculo más fuerte para mantenerse juntos como empresa. 
Finalmente, se encontró que la percepción de unidad y armonía familiar es diferente en México y Colombia. Esto indica una influencia de la cultura, entendida como las prácticas y valores compartidos por un grupo (House, Hanges, Javidan, Dorfman \& Gupta, 2004) que pueden influir en su funcionamiento (Georgas, 1989). Debido a que las prácticas de gestión de las empresas familiares varían en cada país (Gómez, Betancourt y Zapata, 2012; GómezBetancourt, 2010), las diferencias culturales entre las empresa, familiares de México y Colombia deben ser profundizadas en futuros estudios para identificar prácticas nacionales y cómo ellas pueden influir en las dinámicas de este tipo de sociedades.

\section{Conclusiones}

\section{1} a presente investigación buscó identificar las prácticas de gobierno y gestión que más influyen en la unidad y armonía de las empresas familiares, pues mediante un análisis de regresión logística, se identificaron seis prácticas y dos variables de control que influyen en la percepción de la unidad y armonía familiar. La probabilidad de tener una percepción de mejoramiento en la unidad y armonía es más alta cuando: i) la persona que lidera el patrimonio considera necesario el consejo de otros para el buen desarrollo del patrimonio familiar -2.39 veces-; ii) los líderes asisten periódicamente a programas de formación directiva, conferencias y talleres, para el buen desarrollo de sus funciones -2.20 veces-; iii) los actuales y potenciales accionistas fa-miliares tengan los conocimientos necesarios para ejercer un rol responsable como propietarios -1.86 veces-; iv) los propietarios de la familia hacen reuniones adicionales a la asamblea general de accionistas para discutir periódicamente aspectos de la propiedad, analizar información y tomar decisiones -2.26 veces-; y v) los miembros de la familia tienen una estrategia familiar explícita con actividades que fomenten la unidad y armonía, y que les facilite resolver conflictos -3.32 veces-. En contraste, existe mayor probabilidad de tener una percepción de deterioro en la unidady armonía cuando la compañía realiza anualmente una asamblea general de accionistas donde se toman decisiones sobre inversiones, dividendos, endeudamiento, nombramiento de junta directiva, entre otros -.50 veces-. Los hallazgos anteriores dieron sus-tento a la hipótesis planteada y se corroboró que algunas prácticas de gobierno y gestión implementadas en la empresa familiar influyen en su unidad y armonía.

La principal contribución teórica de este estudio es la identificación de prácticas específicas que pueden contribuir o deteriorar las relaciones de la empresa familiar. Se evidenció también, que el efecto de una práctica de gestión en la empresa genera repercusiones en el sistema familiar, razón por la cual las investigaciones con este tipo de compañías deben ser holísticas. Como implicación práctica es preciso señalar que los asesores y las familias empresarias

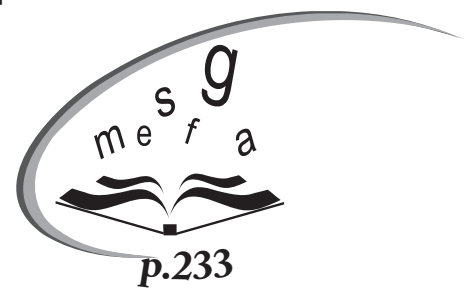
pueden encontrar respuestas en esta investigación para identificar las prácticas presentes en su empresa y proponer planes de acción para corregir aquellas con efectos negativos en el desempeño. 


\section{Limitaciones y recomendaciones para futuras investigaciones}

$\mathrm{U}$ na de las principales limitaciones de este estudio es el muestreo por conveniencia, que puede generar un sesgo debido a que la población presenta un alto nivel de formación y las empresas de la muestra son de un tamaño mayor al promedio de las empresas del país, ya que los encuestados eran, en su mayoría, de pequeñas, medianas y grandes compañías. De esta manera, la generalización de los resultados puede verse afectada y los porcentajes identificados por malas prácticas podrían llegar a ser superiores en empresas de menor tamaño y con directivos menos formados. Una limitación más, es la aproximación al constructo de unidad y armonía, la cual se hace a partir de una sola pregunta con base en la percepción del encuestado; esto puede reflejar una posición individual más que la grupal. Otra limitación fue la dicotomización de las variables, que implica pérdida de calidad en la información y potencia la prescripción del modelo estadístico, sin embargo, para efectos de esta investigación permite presentar el análisis con mayor claridad para el lector.
Esta investigación propone un modelo que contempla 47 prácticas de gobierno y gestión, que fueron inspiradas en la revisión de literatura y la experiencia de los autores, por esto, es posible que otras prácticas deban ser incorporadas en futuras investigaciones para probar su efecto en las variables dependientes. Se sugiere que en investigaciones futuras se examine la influencia de las prácticas en indicadores económicos y no económicos de las empresas, como el crecimiento en ventas, la rentabilidad, el endeudamiento, la innovación, el clima organizacional y la comunicación; y otros de la familia propietaria, como la filantropía y la responsabilidad social. Adicionalmente, se identificó, con este estudio, la necesidad de analizar profundamente la influencia del desempeño económico en las dinámicas de las familias propietarias. Se sugiere también, estudiar el efecto de las prácticas de gobierno y gestión de acuerdo con la generación en que se encuentre la organización, así como los diferentes sistemas que la componen -familia, patrimonio y empresa-. 


\section{Referencias bibliográficas}

Aronoff, C. E., \& Ward, J. L. (2002). Family Business Ownership: How to be an Effective Shareholder. Marietta, GA: Family Enterprise Publishers.

Barbera, F., Bernhard, F., Nacht, J., \& McCann, G. (2015). The relevance of a whole-person learning approach to family business education: Concepts, evidence, and implications. Academy of Management Learning \& Education, 14(3), 322-346.

Basco, R., \& Rodríguez, M. J. P. (2011). Ideal types of family business management: Horizontal fit between family and business decisions and the relationship with family business performance. Journal of Family Business Strategy, 2(3), 151-165.

Gómez-Betancourt, G. G., Betancourt-Ramírez, J. B., \& Zapata-Cuervo, L. N. (2012). Empresas familiares multigeneracionales. Entramado, 8(2), 38-49.

Betancourt, J. (2013). The Influence of the Owners' Council in the Strategy of Families in Business. Unpublished paper.

Betancourt, J., Gómez, G., y López, M. P. (2011). Aproximación a los factores que influyen en la visión del patrimonio en las empresas familiares colombianas. Estudios Gerenciales, 27(120), 165-184.

Botero, I. C., Gomez, G., Betancourt, J. B., \& Lopez, M. P. (2015). Family protocols as governance tools: Understanding why and how family protocols are important in family firms. Journal of Family Business Management, 5(2), 218-237.
Brenes, E. R., Madrigal, K., \& Requena, B. (2011). Corporate governance and family business performance. Journal of Business Research, 64(3), 280-285.

Chandler, A. D. (1962). Strategy and structure: Chapters in the history of the American enterprise. Cambridge: Massachusetts Institute of Technology.

Chrisman, J. J., Chua, J. H., \& Litz, R. A. (2004). Comparing the agency costs of family and nonfamily firms: Conceptual issues and exploratory evidence. Entrepreneurship Theory and Practice, 28(4), 335-354.

Corbella, R. J. (1994). Descubrir la psicología. Percepción. Folio, 1, Barcelona: Ed. Folio.

Daily, C. M., \& Dalton, D. R. (1993). Board of directors leadership and structure: Control and performance implications. Entrepreneurship: Theory and Practice, 17(3), 65-82.

Fiegener, M. K., Brown, B. M., Prince, R. A., \& File, K. M. (1996). Passing on strategic vision. Journal of Small Business Management, 34(3), 15.

Galbraith, J. R. (1995). Designing organizations: An executive briefing on strategy, structure, and process. Jossey-Bass.

Gallo, M. A. (1995). Empresa familiar: texto y casos. Barcelona: Praxis.

Georgas, J. (1989). Changing family values in Greece from collectivist to individualist. Journal of Cross-Cultural Psychology, 20(1), pp. 80-91. 
Gómez, G. (2000). Tipologías de empresas familiares: un marco conceptual basado en los factores de la dirección estratégica y la confianza. Tesis doctoral no publicada, IESE, Navarra, España: Universidad de Navarra.

Gómez-Betancourt, G. (2005). ¿Son iguales todas las empresas familiares?: caminos por recorrer. Bogotá: Grupo Editorial Norma.

Gómez-Betancourt, G. (2010). ¿Cómo construir un legado familiar?: un modelo para tener familias empresarias perdurables. CENGAGE.

Gómez-Betancourt, G., y Zapata-Cuervo, N. (2013). Gobierno corporativo: una comparación de códigos de gobierno en el mundo, un modelo para empresas latinoaméricanas familiares y no familiares. Entramado, 9(2), 98-117.

Grant, R. M. (2016). Contemporary strategy analysis: Text and cases edition. John Wiley \& Sons.

Habbershon, T. G., \& Astrachan, J. H. (1997). Research note perceptions are reality: How family meetings lead to collective action. Family Business Review, 10(1), 37-52.

Harvey, M., \& Evans, R. E. (1994). Family business and multiple levels of conflict. Family Business Review, 7(4), 331-348.

Hoekman, D. (1999). Succession planning is must for continued success. Grand Rapids Business Journal, 17(43), B3-4.

House, R. J., Hanges, P. J., Javidan, M., Dorfman, P. W., \& Gupta, V. (2004). Culture, leadership, and organizations: The GLOBE study of 62 societies. Sage publications.
Jaffe, D. T., \& Lane, S. H. (2004). Sustaining a family dynasty: key issues facing complex multigenerational businessand investmentowning families. Family Business Review, 17(1), 81-98.

Jehn, K. A. (1995). A multimethod examination of the benefits and detriments of intragroup conflict. Administrative Science Quarterly, pp. 256-282.

Jiménez, M. C. R., Martos, M. C. V., y Jiménez, R. M. (2015). Organisational harmony as a value in family businesses and its influence on performance. Journal of Business Ethics, 126(2), pp. 259-272.

Jussila, I. (2006). A Framework for Analyzing Managers' Self-serving and Cooperative Behaviors. Docuestudio de casos colombianos. Estudios Gerenciales, 24(109), 3763.

Kellermanns, F. W., \& Eddleston, K. A. (2007). A family perspective on when conflict benefits family firm performance. Journal of Business Research, 60(10), pp.1048-1057.

Lane, S., Astrachan, J., Keyt, A., \& McMillan, K. (2006). Guidelines for family business boards of directors. Family Business Review, 19(2), pp. 147-167.

Lansberg, I. (1999). Succeeding generations: Realizing the dream of families in business. Harvard Business Press.

Lave, J., \& Wenger, E. (1991). Situated learning: Legitimate peripheral participation. Cambridge University Press.

Lester, R. H., \& Cannella, A. A. (2006). Interorganizational Familiness: How Family 
Firms Use Interlocking Directorates to Build Communityevel Social Capital 1. Entrepreneurship Theory and Practice, 30(6), pp. 755-775.

Li, C. (2006). The Confucian ideal of harmony. Philosophy East and West, 56(4), 583-603.

Londoño, J. L. (1996). Violencia, psiquis y capital social. Revista Consigna, 20(450), pp. 7-8.

Mora y Araújo, M. (2012). El poder de la conversación: elementos para una teoría de la opinión pública. La Crujía.

Olson, P. D., Zuiker, V. S., Danes, S. M., Stafford, K., Heck, R. K. Z., \& Duncan, K. A. (2003). The impact of the family and the business on family business sustainability. Journal of Business Venturing, 18(5), 639-666.

Peterson, P., \& Distelberg, B. J. (2011). Differentiating value orientations and unity in values as predictors of varying family business system processes. Journal of Family Business Strategy, 2(4), pp. 207-219.

Pieper, T. M., Klein, S. B., \& Jaskiewicz, P. (2008). The Impact of Goal Alignment on Board Existence and Top Management Team Composition: Evidence from Family Influenced Businesses. Journal of Small
Business Management, 46(3), pp. 372-394.

Schulze, W. S., Lubatkin, M. H., Dino, R. N., \& Buchholtz, A. K. (2001). Agency relationships in family firms: Theory and evidence. Organization Science, 12(2), pp.99-116.

Sirmon, D. G., \& Hitt, M. A. (2003). Managing resources: Linking unique resources, management, and wealth creation in family firms. Entrepreneurship Theory and Practice, 27(4), pp. 339-358.

Suess, J. (2014). Family governance-Literature review and the development of a conceptual model. Journal of Family Business Strategy, 5(2), pp. 138-155.

Tagiuri, R., \& Davis, J. A. (1992). On the goals of successful family companies. Family Business Review, 5(1), pp. 43-62.

Ward, J. L. (2011). Keeping the family business healthy: How to plan for continuing growth, profitability, and family leadership. Palgrave Macmillan.

Zahra, S. A. (2003). International expansion of US manufacturing family businesses: The effect of ownership and involvement. Journal of Business Venturing, 18(4), pp. 495-512. 